\title{
Digital Image Watermarking using SWT and SVD
}

\author{
Manju Yadav', Ashok Kumar ${ }^{2}$ \\ M.Tech, Electronics Department, Kamla Nehru Inst of Technology, Sultanpur, Uttar Pradesh, India ${ }^{1}$ \\ Asst. Professor, Electronics Department, Kamla Nehru Inst of Technology, Sultanpur, Uttar Pradesh, India ${ }^{2}$
}

\begin{abstract}
In this tech-era everyone is using social networking sites and many messenger sites. Using internet anyone can send data and access any data in few seconds in all over world but the problem is this if any data once send over a network lose its privacy any third party can access your data and can use your data in illegitimate manner and you cannot claim that this data belongs to you but if you put a watermark in that data the third party cannot access your data if your data send to any wrong person by mistaken then that person can send back your data by identifying watermark. So now main objective of our paper is to make a compatible relation between imperceptibility and robustness. .In this paper an improved technique based on SWT and SVD is presented that has good PSNR, MSE and NCC compare to other technique.
\end{abstract}

Keywords: SWT (Stationary wavelet transform), SVD, PSNR, NCC.

\section{I.INTRODUCTION}

In this digital world, there is rapidly growing and sharing of multimedia files. Billions of digital files are transferred via the internet per day. There are many advantages privileged to users. But there are numerous disadvantages also like a security problem, copyright of data, data transformation. The main source of these problems is internet. So security of the authentic information and some other issues has become a big question with multimedia source and content. Digital data can be easily copied and transferred to another user without loss of data and quality of data. Solving these types of problems are challenges for the researchers .Data hiding technique is the solution of these problems. Some multimedia data is introduced into the owner's data; further to prove the owner's data extracted process is used. This idea was initially used in bank currency notes. The watermark is embedded in the currency notes to hide the originality of notes. Digital Watermarking technique can be classified by many different ways i.e. according to the domain, the type of document, human perception, and the application. Based on the embedding information concept, watermarking algorithms can be classified as either spatial or transform domain. In the spatial domain, the watermark information is directly embedded in the pixel value of the host or cover image. These methods are fast and simple and also provide high capacity for embedding watermarks. Spatial domain methods may have some advantages and may overcome cropping attacks, but their main drawback is their weaknesses against noise or lossy compression attacks. In addition, upon discovering the method, embedded watermarks can easily be modified by a third party. In the transform domain, we get the watermarked image by embedding the watermark into the transformed version of the original image.

According to human perception, the watermarking methods can be grouped into visible and invisible watermarks. A visible watermark is logos, and can perceive by the human eye that's why visible watermark put at the corners of images for copyright protection for example most of T.V. channel use their logo for their content protection. Now other side Invisible watermarks are hidden cannot perceived by the human eyes and used for application such as authentication, integrity verification and copyright protection.On the basis of some criteria Invisible watermarking methods can be divided into four groups: fragile, semi fragile, robust, and hybrid methods. In fragile method watermark easily be destroyed by the smallest of modifications. Robust digital imagewatermarking method isused for copyright protection purpose, should be resistant against multiple different attacks. The robustness of these methods can be calculated by applying different attacks on the watermarked images and comparing the embedded and extracted watermark. Hybrid digital image watermarking is a new technique that is based on robust and fragile techniques this hybrid watermarking technique provides authentication, integrity verification, and copyright protection simultaneously. In addition to all above discussion reversibility is another important aspect in watermarking. Compared to the other watermarking technique, reversible data hiding is the best technique it not only stores the watermark but also the original multimedia perfectly, which is a main requirement for medical and military applications. The property of reversible methods is the ability to recover the original image without any distortion after extracting the watermark bits, besides providing tamper proofing and authentication. Using a reversible data hiding algorithm by embedding patient details and diagnostics data into the medical image, medical officers can recover perfectly both the hidden information as well as the image itself. 


\section{II.PROPOSED WORK}

A highly robust algorithm is proposed for watermarking using SWT and SVD techniques. By applying 2D-SWT, the host image is fragmented into four sub-bands like LL, LH, HL, and HH. Then SVD is applied to one of the four subbands. Similarly 2D-SWT is applied to the watermark image and then SVD is applied to one of the frequency subbands obtained in the above process. The singular matrix of watermark image is then added to the singular matrix of the host image with suitable scale factor to get watermarked image.

\section{A. Watermark Embedding}

It is the process of inserting watermark into an image.

1) Perform SWT on original image to get frequency sub-bands LL1', LH1', HL1', HH1' again perform SWT on LL1' and get sub-bands LL1", LH1", HL1", HH1'".

2) Select one of the frequency sub-bands, Here HL1" sub-band is selected.

3) Divide the HL1" sub-band into S1, V1and U1 using SVD and obtain singular matrix.

$\mathrm{HL} 1$ " $=\mathrm{U} 1 * \mathrm{~S} 1 * \mathrm{~V} 1{ }^{\mathrm{T}}$

4) Perform SWT on watermark image to get frequency sub-bands LL2', LH2', HL2', HH2' again perform SWT on LL2' and get sub-bands LL2", LH2", HL2", HH2'”.

5) Select one of the sub-bands. Here HL2"' sub-band is selected.

6) Apply SVD to HL2"' and obtain singular matrix of each sub-band using Eq.

$\mathrm{HL} 2 "=\mathrm{U} 2 * \mathrm{~S} 2 * \mathrm{~V} 2{ }^{\mathrm{T}}$

Where $\mathrm{U} 2, \mathrm{~S} 2, \mathrm{~V} 2$ are matrices.

7) Add singular matrix values of host image with singular matrix values of watermark image multiplied by scaling factor using Eq. .

$\mathrm{S}=\mathrm{S} 1+\mathrm{k} * \mathrm{~S} 2 \quad$ Where $\mathrm{k}=$ scaling factor, it controls the strength of the watermark inserted.

10) Combine U1, S and V1 to get HL1' using Eq.

HL1' $($ modified $)=$ U1SV1 ${ }^{\mathrm{T}}$

12) Apply 2- Level ISWT on host image sub-bands by replacing original HL1' sub-band by modified HL1' sub-band to get watermarked image.

\section{B. Watermark Extraction}

The following steps are employed for extracting the watermark from watermarked image.

1) Perform 2D-SWT on watermarked image.

2) Select HL frequency sub-band (frequency sub-band that contains watermark in it).

4) Apply SVD to that frequency band and obtain singular matrix.

$$
\mathrm{HL} '=\mathrm{U} * \mathrm{~S} * \mathrm{~V}^{\mathrm{T}}
$$

5) Compute the singular matrix value of watermark by the use of given equation.

$$
\mathrm{S} 2=(\mathrm{S}-\mathrm{S} 1) / \mathrm{k}
$$

6) Calculate HL2' using ISVD (by combining U2, S2, V2)

$$
\text { HL2' (modified) }=\mathrm{U} 2 * \mathrm{~S} 2 * \mathrm{~V} 2^{\mathrm{T}}
$$

7) Apply 2 Level-ISWT to the watermark image by replacing the original HL2'sub-band with the above modified sub band to get extracted watermark image.

\section{REFERENCE IMAGES}

These images are taken to embed the watermark downloaded from standard database.Overall analysis done with the 512x512 image .Here images used that are downloaded from USC-SIPI image database for watermarking algorithms MATLAB software used for simulation of this technique.

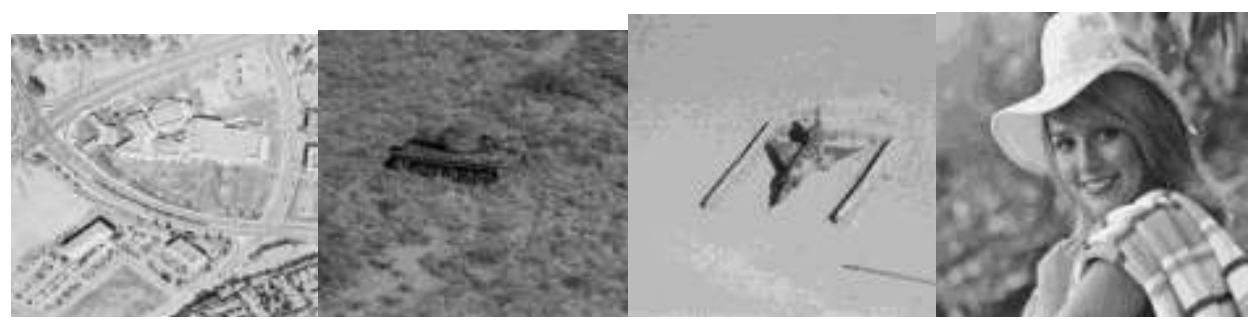

Fig-1 Reference image (a) Pentagon (b) War tank(c) Aircraft (d) Girl 


\section{WATERMARK IMAGE}

These images are used to embed in the reference image for security purpose.
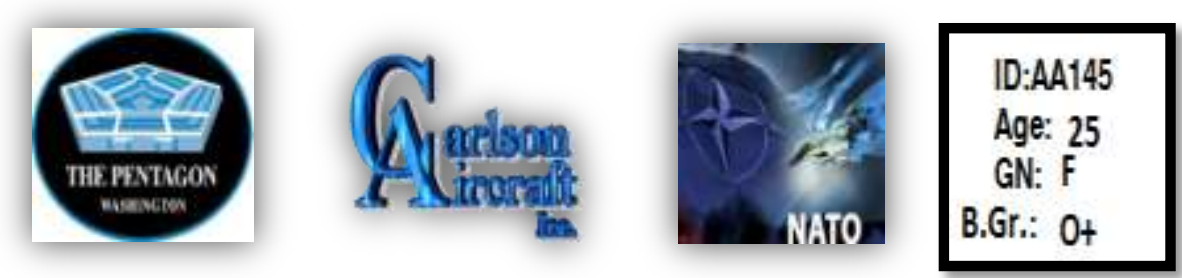

Fig -2 Watermark image (a) Pentagon logo (b) Aircraft company logo (c) Nato logo (d) Identity details logo

\section{WATERMARKED IMAGE}

After embedding the watermark in to the reference image watermarked image obtained. Watermark hidden in the reference image is robust not degraded the quality of real image. Watermarked image looks like reference image means watermark is imperceptible here. Image quality is determined by calculating PSNR after comparing the real image with the watermarked image.
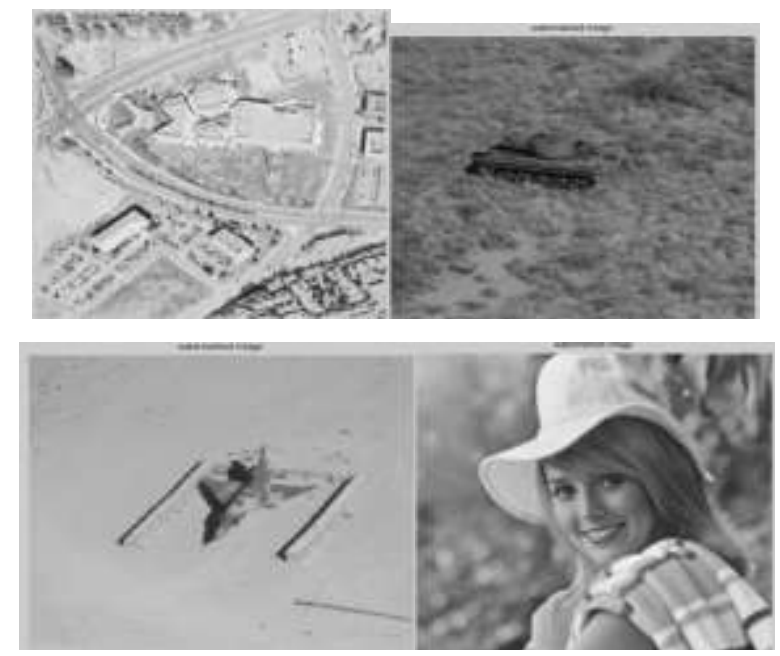

Fig-3 Watermarked image (a) Pentagon (b) War tank(c) Aircraft (d) Girl

\section{EXTRACTED WATERMARK}

Finally watermark extracted from real image and that watermark compare with the real one to detect any attack and by this comparison we obtain the normalised correlation coefficient (NCC) Calculating NCC we detect the robustness of watermark.

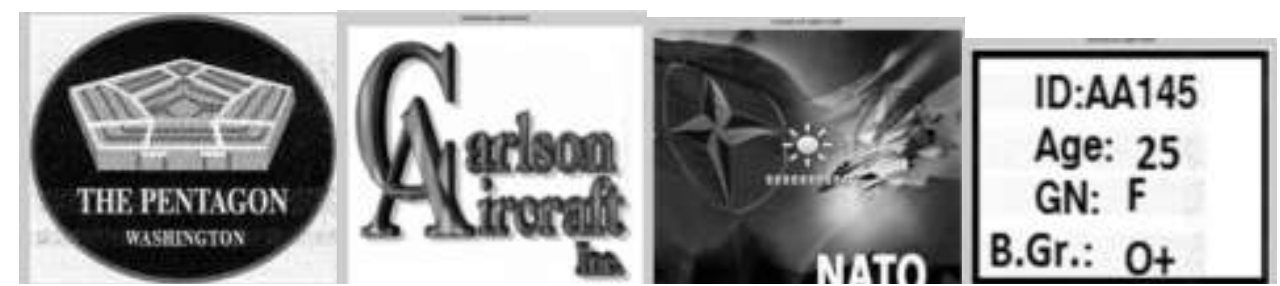

Fig-4 Extracted Watermark (a) Pentagon logo (b) Aircraft company logo (c) Nato logo (d) Identity details logo

IX RESULTS

\section{A. Mean Square Error (MSE)}

Mean square is an important parameter that calculated by comparing real image with the watermarked image MSE should be low to get better quality of watermarked image. Using the given formula we calculate MSE 
Table-1 PSNR for SWT-SVD Based Watermarking

\begin{tabular}{|l|l|l|l|l|}
\hline Visibility factor & PSNR(Aircraft) & PSNR(Girl) & PSNR(Pentagon) & PSNR( Tank) \\
\hline $\mathbf{0 . 0 4}$ & $\mathbf{5 6 . 9 6 2 7}$ & $\mathbf{5 3 . 0 0 6}$ & $\mathbf{4 6 . 0 6 1 1}$ & $\mathbf{5 1 . 3 4 5}$ \\
\hline $\mathbf{0 . 0 3}$ & $\mathbf{5 7 . 0 5 9 2}$ & $\mathbf{5 3 . 1 2 8}$ & $\mathbf{4 6 . 1 1 3 6}$ & $\mathbf{5 1 . 3 7 8 6}$ \\
\hline $\mathbf{0 . 0 2 5}$ & $\mathbf{5 7 . 1 0 9 6}$ & $\mathbf{5 3 . 1 7 6}$ & $\mathbf{4 6 . 1 3 8 1}$ & $\mathbf{5 1 . 4 0 2 3}$ \\
\hline $\mathbf{0 . 0 2}$ & $\mathbf{5 7 . 1 6 3 5}$ & $\mathbf{5 3 . 2 3 8}$ & $\mathbf{4 6 . 1 6 1 6}$ & $\mathbf{5 1 . 4 2 4 4}$ \\
\hline $\mathbf{0 . 0 1}$ & $\mathbf{5 7 . 2 6 3 1}$ & $\mathbf{5 3 . 3 4 6}$ & $\mathbf{4 6 . 2 1 1 6}$ & $\mathbf{5 1 . 4 6 6 5}$ \\
\hline $\mathbf{0 . 0 0 5}$ & $\mathbf{5 7 . 3 1 4 2}$ & $\mathbf{5 3 . 3 9 8}$ & $\mathbf{4 6 . 2 3 5 7}$ & $\mathbf{5 1 . 4 8 8 1}$ \\
\hline
\end{tabular}

$M S E=\frac{1}{M N} \sum_{x=0}^{M-1} \sum_{y=0}^{N-1}\left(I(x, y)-I^{\prime}(x, y)\right)^{2}$

\section{B. Peak Signal to Noise Ratio (PSNR)}

PSNR indicates the visibility of noise in an image. $P S N R=10 \log \frac{\max ^{2}}{M S E}$

\section{Normalized Correlation Coefficient (NCC)}

The correlation between original watermarks that is embedded with the extracted watermark is computed with NCC. It reveals the watermark that was embedded, that's why NCC is called as measure of authenticity. NCC value should lie between -1 to 1 .

\section{Graphs between Different Parameter}

Here PSNR for different image is calculated on different visibility factor for SWT and SVD based Watermarking. As visibility factor reduces the PSNR increases.

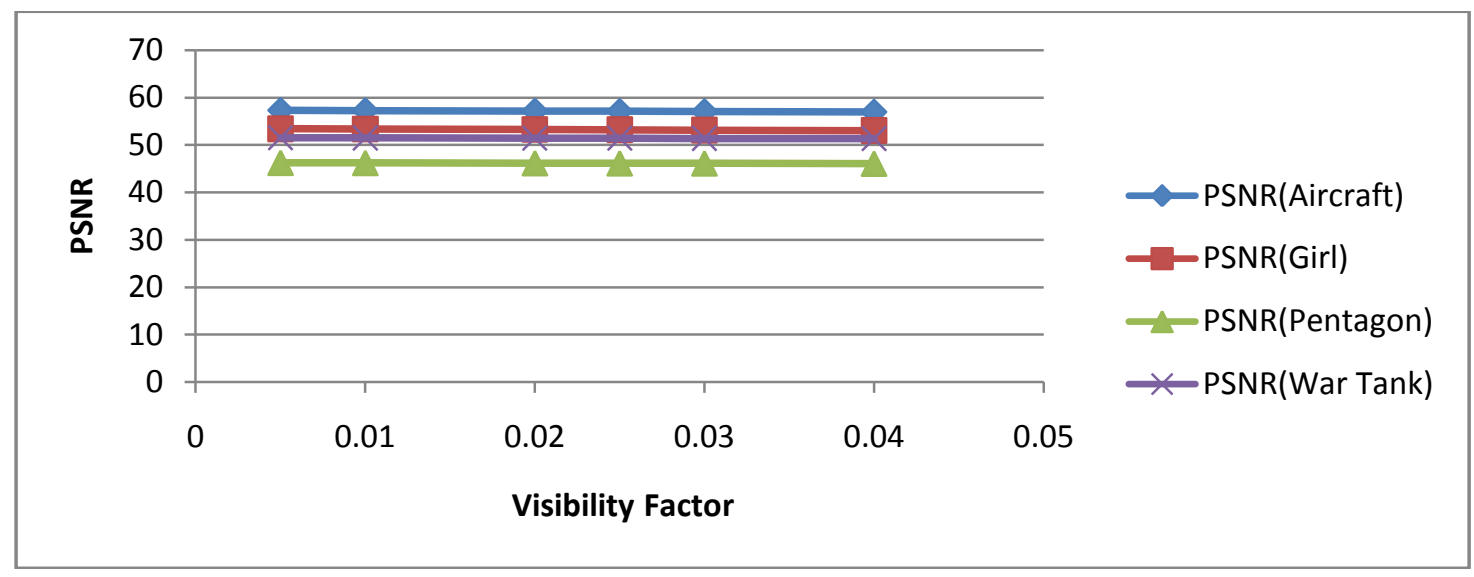

Fig-5 Graph between PSNR and Visibility factor

Table -2 NCC (Normalized Correlation Coefficient) for SWT-SVD Based Watermarking

\begin{tabular}{|c|c|c|c|c|}
\hline Visibility factor & $\begin{array}{l}\text { CC(Aircraft logo } \\
\text { as a watermark ) }\end{array}$ & $\begin{array}{l}\text { CC(Identity as a } \\
\text { watermark ) }\end{array}$ & $\begin{array}{l}\text { CC(Pentagon as a } \\
\text { watermark) }\end{array}$ & $\begin{array}{l}\text { CC (Nato as } \\
\text { watermark ) }\end{array}$ \\
\hline 0.04 & $\mathbf{0 . 9 9 9 8}$ & $\mathbf{0 . 9 9 9 6}$ & $\mathbf{0 . 9 9 9 2}$ & $\mathbf{0 . 9 9 9 3}$ \\
\hline 0.03 & $\mathbf{0 . 9 9 9 7}$ & $\mathbf{0 . 9 9 9 5}$ & $\mathbf{0 . 9 9 8 9}$ & $\mathbf{0 . 9 9 8 7}$ \\
\hline $\mathbf{0 . 0 2 5}$ & $\mathbf{0 . 9 9 9 4}$ & $\mathbf{0 . 9 9 9 0}$ & $\mathbf{0 . 9 9 8 6}$ & $\mathbf{0 . 9 9 8 1}$ \\
\hline $\mathbf{0 . 0 2}$ & $\mathbf{0 . 9 9 9 0}$ & $\mathbf{0 . 9 9 8 9}$ & $\mathbf{0 . 9 9 7 7}$ & $\mathbf{0 . 9 9 7 2}$ \\
\hline 0.01 & $\mathbf{0 . 9 9 8 4}$ & $\mathbf{0 . 9 9 8 6}$ & $\mathbf{0 . 9 9 7 2}$ & $\mathbf{0 . 9 9 0 8}$ \\
\hline $\mathbf{0 . 0 0 5}$ & $\mathbf{0 . 9 9 6 0}$ & $\mathbf{0 . 9 9 8 4}$ & $\mathbf{0 . 9 9 6 7}$ & $\mathbf{0 . 9 6 5 9}$ \\
\hline
\end{tabular}

Here NCC for different image is calculated on different visibility factor for SWT and SVD based Watermarking. As visibility factor reduces the NCC decreases and PSNR improves Visibility factor shows the effect of watermark in the reference image. 


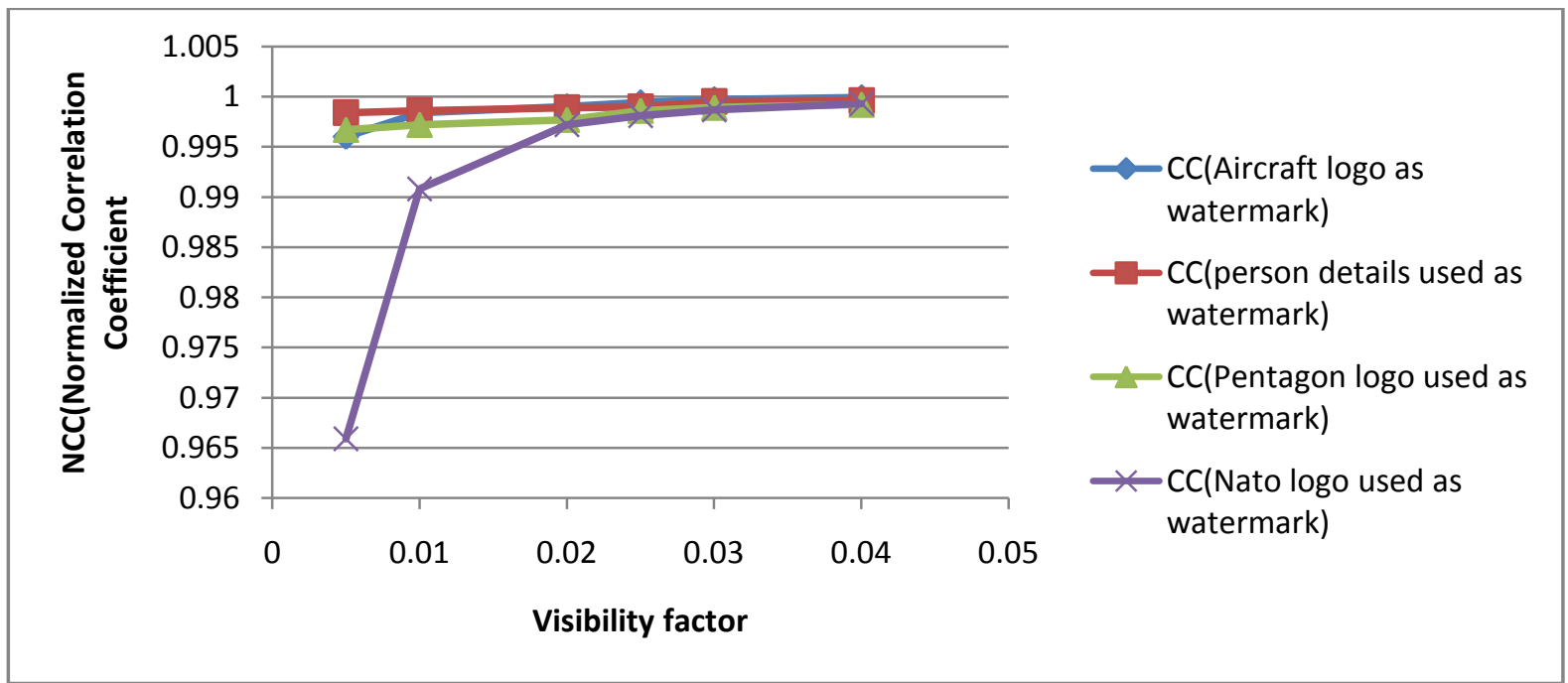

Fig-6 Graph between NCC and Visibility factor for different image for SWT-SVD based watermarking

From the above table and graph we can see how NCC varies according the visibility factor.

\section{X.CONCLUSION}

Digital watermarking proved to be an important technique for copyright protection and deterrence to multimedia Piracy This watermarking technique gives better result than other technique .In this paper, variable visibility factor is used for the insertion of watermark into the low frequency component of the host image. Simulation results show that the feature of the watermarked image and the recoveredwatermark are dependent only on the visibility factors and also show that the SWT and SVD give superior results than 2-level DWT [1].

\section{REFERENCES}

[1]Rita Chaudhary, Girish Parimar',A Robust image Watermarking Technique using 2-level Discrete Wavelet Transform (DWT)' In IEEE 2nd International Conference on Communication, Control and Intelligent Systems (CCIS).

[2]"Digital Image Processing Using Matlab"by Rafael Gonzalez, Richard E.Woods,Steven L.Eddins.

[3]Monika Patel, Preeti Srinivas ,Sajja, Jigar Patel "Enhancement of DWT based Watermarking Technique for Images"in International Journal of Advanced Research in Computer and Communication Engineering Vol. 2, Issue 12, December 2013.

[4]https://en.wikipedia.org/wiki/Stationary_wavelet_transform.

[5]Sridevi and Sameen Fatima "digital image watermarking using genetic algorithm inDWT and SVD transform" in 3 rd international conference on computer intelligence and information technology at pp 485-490.

[6] P.V.Nagarjuna, K.Ranjeet "Robust blind digital image watermarking scheme based on Stationry Wavelet Transform"in 2013 sixth international conference on contemporary computing at pp 451-454.

[7]Mohamed Radouane,Rochdi Messoussi,Raja Touahni,Tarik Boujiha "Robust Method of Digital Image Watermarking using SVD Transform on DWT Coefficients with Optimal Block"in 2014 International Conference on Multimedia Computing and System at pp255-260.

[8] Tulika Bhuvan ,Vinay Kumar Shrivastava,Falgun Thakkar"Shuffled SVD based robust and secure digital image watermarking"in International Conference on Electrical, Electronics, and Optimization Techniques (ICEEOT) - 2016 pp 1229-1233.

[9] Ms. Nazish Fatima,D.J.Tuptewar "comparison of hybrid watermarking technique on different colour spaces" in 2016 Conference on Advances in Signal Processing (CASP)Cummins College of Engineering for Women, Pune Jun 9-11, 2016. 